\title{
WEIGHTED COMPOSITION OPERATORS ON GENERALIZED FOCK-TYPE SPACES
}

\section{WALEED AL-RAWASHDEH}

Department of Mathematical Sciences

Montana Tech of the University of Montana

1300 West Park Street

Butte, Montana 59701

USA

e-mail: walrawashdeh@mtech.edu

\begin{abstract}
Let $\psi$ be an entire self-map of the $n$-dimensional Euclidean complex space $\mathbb{C}^{n}$ and $u$ be an entire function on $\mathbb{C}^{n}$. A weighted composition operator induced by $\psi$ with weight $u$ is given by $\left(u C_{\psi} f\right)(z)=u(z) f(\psi(z))$, for $z \in \mathbb{C}^{n}$ and $f$ is entire function on $\mathbb{C}^{n}$. In this paper, we study weighted composition operators act between generalized Fock-types spaces. We characterize the boundedness and compactness of these operators act between $\mathcal{F}_{\phi}^{p}\left(\right.$ or $\left.\mathcal{F}_{\phi}^{\infty, 0}\right)$ and $\mathcal{F}_{\phi}^{\infty}\left(\right.$ or $\left.\mathcal{F}_{\phi}^{\infty, 0}\right)$ when $0<p \leq \infty$.
\end{abstract}

2010 Mathematics Subject Classification: Primary 47B38, 30D15, 44A20; Secondary 30C40, 30D20, 44A15.

Keywords and phrases: weighted composition operators, bounded operators, compact operators, entire functions, generalized Fock-type spaces.

Received April 7, 2015

(C) 2015 Scientific Advances Publishers 


\section{Introduction}

Let $\mathbb{C}^{n}$ be the $n$-dimensional complex Euclidean space and $d v$ be the usual Lebesgue volume measure on $\mathbb{C}^{n}$. Throughout this paper, we assume that $\phi \in C^{2}\left(\mathbb{C}^{n}\right)$ is a given real valued function on $\mathbb{C}^{n}$ such that

$$
m w_{0}<d d^{c} \phi<M w_{0}
$$

holds uniformly pointwise on $\mathbb{C}^{n}$ for some positive constants $m$ and $M$, where $d$ is the usual exterior derivative, $d^{c}=\frac{i}{4}(\bar{\partial}-\partial)$, and $w_{0}=d d^{c}|\cdot|^{2}$ is the standard Euclidean Kähler form on $\mathbb{C}^{n}$. For $0<p<\infty$, the generalized Fock space $\mathcal{F}_{\phi}^{p}\left(\mathbb{C}^{n}\right)$ consists of all entire functions $f$ on $\mathbb{C}^{n}$ such that

$$
\|f\|_{p, \phi}^{p}=\int_{\mathbb{C}^{n}}|f(z)|^{p} e^{-p \phi(z)} d v(z)
$$

is finite. Let $\mathcal{H}\left(\mathbb{C}^{n}\right)$ be the space of all entire functions on $\mathbb{C}^{n}$. The generalized Fock-type spaces $\mathcal{F}_{\phi}^{\infty}\left(\mathbb{C}^{n}\right)$ and $\mathcal{F}_{\phi}^{\infty, 0}\left(\mathbb{C}^{n}\right)$ are defined as

$$
\begin{aligned}
& \mathcal{F}_{\phi}^{\infty}=\left\{f \in \mathcal{H}\left(\mathbb{C}^{n}\right):\|f\|_{\infty, \phi}=\sup _{z \in \mathbb{C}^{n}}|f(z)| e^{-\phi(z)}<\infty\right\}, \\
& \mathcal{F}_{\phi}^{\infty, 0}=\left\{f \in \mathcal{F}_{\phi}^{\infty}:\|f\|_{\infty, 0, \phi}=\lim _{|z| \rightarrow \infty}|f(z)| e^{-\phi(z)}=0\right\} .
\end{aligned}
$$

For $\alpha>0$ and $0<p \leq \infty$, if $\phi(z)=\frac{\alpha}{2}|z|^{2}$, we get the weighted Fock spaces $\mathcal{F}_{\alpha}^{p}$. For the overview of the studies on $\mathcal{F}_{\alpha}^{p}$, we refer to the monographs [16] and [20]. If $\phi(z)=\frac{|z|^{2}}{2}-m \log |z|$, where $m$ is a nonnegative integer we get the Fock-Sobolev spaces $\mathcal{F}^{p, m}$, these spaces were first introduced by Cho and Zhu in [2]. 
It is well-known that, for $1 \leq p \leq \infty, \mathcal{F}_{\phi}^{p}$ is a Banach space under the norm $\|\cdot\|_{p, \phi}$. Also, for $0<p<1$, the space $\mathcal{F}_{\phi}^{p}$ is an $F$-space under $d(f, g)=\|f-g\|_{p, \phi}^{p}$. Moreover, the Hilbert space $\mathcal{F}_{\phi}^{2}$ is closed subspace of $L^{2}\left(\mathbb{C}^{n}, e^{-2 \phi} d v\right)$ with the Bergman kernel $K_{\phi}(\cdot, \cdot)$. The Bergman orthogonal projection $P: L^{2}\left(\mathbb{C}^{n}, e^{-2 \phi} d v\right) \rightarrow \mathcal{F}_{\phi}^{2}$ is given by integrating against a kernel $K_{\phi}(w, z)=K_{\phi, z}(w)$ and by usual Hilbert space theory; that is,

$$
\operatorname{Pf}(z)=\int_{\mathbb{C}^{n}} K_{\phi}(z, w) f(w) e^{-2 \phi(w)} d v(w)
$$

For any $z \in \mathbb{C}^{n}$ and $r>0$, we use

$$
B(z, r)=\left\{w \in \mathbb{C}^{n}:|w-z|<r\right\}
$$

to denote the Euclidean ball centered at $z$ with radius $r$. It is known that, see [3] for $n=1$ and [7] for $n \geq 2$, there are positive constants $\theta$ and $C$ such that for any $z, w \in \mathbb{C}^{n}$

$$
\left|K_{\phi}(z, w)\right| e^{-\phi(z)} e^{-\phi(w)} \leq C e^{-\theta|z-w|} .
$$

Using Proposition 3.3 of [13], there are positive constants $r, C_{1}$, and $C_{2}$ such that for each $z \in \mathbb{C}^{n}$ and each $w \in B(z, r)$, we have

$$
\left|K_{\phi}(z, w)\right| e^{-\phi(z)} e^{-\phi(w)} \geq C_{1}\left|K_{\phi}(z, z)\right| e^{-2 \phi(z)} \geq C_{2} .
$$

Using the previous inequalities (1) and (2), it is easy to see that for some positive constants $C_{3}$ and $C_{4}$, we have

$$
C_{3} \leq\left\|K_{\phi, z} e^{-\phi(z)}\right\|_{p, \phi} \leq C_{4}
$$


Suppose $\psi$ is an entire function maps $\mathbb{C}^{n}$ into itself and $u$ is an entire function on $\mathbb{C}^{n}$, the weighted composition operator $u C_{\psi}$ is defined on the space $\mathcal{H}\left(\mathbb{C}^{n}\right)$ of all entire functions on $\mathbb{C}^{n}$ by

$$
\left(u C_{\psi} f\right)(z)=u(z) f(\psi(z))
$$

for all $f \in \mathcal{H}\left(\mathbb{C}^{n}\right)$ and $z \in \mathbb{C}^{n}$. The composition operator $C_{\psi}$ is a weighted composition operator with the weight function $u$ identically equal to 1 . It is well-known that the composition operator $C_{\psi} f=f \circ \psi$ defines a linear operator $C_{\psi}$, which acts boundedly on spaces of entire functions on $\mathbb{C}^{n}$.

These operators have been studied on many spaces of analytic functions and entire functions as well. During the past few decades, much effort has been devoted to the study of these operators with the goal of explaining the operator-theoretic properties of $u C_{\psi}$ in terms of the function-theoretic properties of the induced maps $u$ and $\psi$. On the spaces of analytic functions, we refer to the monographs [4], [8], [9], [14], [21], and [22] for the overview of the field as of the early 1990s. See [16] and [20] for the studies on the spaces of entire functions.

Recently, boundedness and compactness of (weighted) composition operators on Fock spaces have been studied by the authors of these papers [1], [11], [12], [15], [17], [18], and [19]. The authors of those papers used classical techniques which were used by many authors in Bergman and Hardy spaces, see, for example, [4], [5], [6], [10], [14], [21], and [22]. In this paper, we use similar techniques to characterize the boundedness and compactness of these operators acting between Fock-type spaces $\mathcal{F}_{\phi}^{p}\left(\right.$ or $\left.\mathcal{F}_{\phi}^{\infty, 0}\right)$ and $\mathcal{F}_{\phi}^{\infty}\left(\right.$ or $\left.\mathcal{F}_{\phi}^{\infty, 0}\right)$ for $0<p \leq \infty$. The results in this paper are generalizations of the previous ones; in fact, if $\phi(z)=\frac{\alpha}{2}|z|^{2}$, for 
$\alpha>0$, we get the results in the papers [15], [17], and [18]. If $\phi(z)=\frac{\alpha}{2}|z|^{2}-m \log |z|$, where $m$ is a non-negative integer we get the results in the paper [11].

The following lemma is a basic fact about the compactness of weighted composition operators. The lemma was proved in a standard way in many references, see, for example, (Proposition 3.11, [4]) in the context of analytic function spaces and (Proposition 4.3, [13]) in the context of entire function spaces. Thus, we omit the trivial proof's details.

Lemma 1.1. For $0<p, q<\infty$ and $d d^{c} \phi \simeq \omega_{0}$. Let $\psi$ be an entire self-map of $\mathbb{C}^{n}$ and $u$ is an entire function of $\mathbb{C}^{n}$ such that $u C_{\psi}$ is bounded from $\mathcal{F}_{\phi}^{p}$ into $\mathcal{F}_{\phi}^{q}$. Then $u C_{\psi}$ is compact if and only if whenever $\left\{f_{n}\right\}$ is bounded sequence in $\mathcal{F}_{\phi}^{p}$ and $f_{n} \rightarrow 0$ uniformly on compact subsets of $\mathbb{C}^{n}$, then $\left\|u C_{\psi}\left(f_{n}\right)\right\|_{q, \phi} \rightarrow 0$.

Remark 1.2. Using Proposition 2.3 of [13], for any entire function $f$ and some positive constant $C$, we have

$$
\begin{aligned}
|f(z)|^{p} e^{-p \phi(z)} & \leq C \int_{B(z, r)}|f(w)|^{p} e^{-p \phi(w)} d v(w) \\
& \leq C \int_{\mathbb{C}^{n}}|f(w)|^{p} e^{-p \phi(w)} d v(w) .
\end{aligned}
$$

This gives $\mathcal{F}_{\phi}^{p} \subset \mathcal{F}_{\phi}^{\infty, 0} \subset \mathcal{F}_{\phi}^{\infty}$. Hence, the previous Lemma 1.1 holds if we replace $\mathcal{F}_{\phi}^{p}$ and $\mathcal{F}_{\phi}^{q}$ with larger spaces.

\section{Main Results}

In this section, we characterize boundedness and compactness of the weighted composition operator $u C_{\psi}$ that acts between $\mathcal{F}_{\phi}^{p}\left(\right.$ or $\left.\mathcal{F}_{\phi}^{\infty, 0}\right)$ and $\mathcal{F}_{\phi}^{\infty}\left(\right.$ or $\left.\mathcal{F}_{\phi}^{\infty, 0}\right)$. 
Theorem 2.1. Let $0<p \leq \infty$, let $\psi$ be an entire self-map of $\mathbb{C}^{n}$, and let $u$ be an entire function of $\mathbb{C}^{n}$. Then the operator $u C_{\psi}: \mathcal{F}_{\phi}^{p} \rightarrow \mathcal{F}_{\phi}^{\infty}$ is bounded if and only if

$$
\sup _{z \in \mathbb{C}^{n}}|u(z)| e^{\phi(\psi(z))} e^{-\phi(z)}
$$

is finite. Moreover, if $u C_{\psi}: \mathcal{F}_{\phi}^{p} \rightarrow \mathcal{F}_{\phi}^{\infty}$ is bounded, then there exist two positive constants $C_{1}$ and $C_{2}$ such that

$$
C_{2}\left\||u(z)| e^{\phi(\psi(z))} e^{-\phi(z)}\right\|_{L^{\infty}} \leq\left\|u C_{\psi}\right\|_{\infty, \phi} \leq C_{1}\left\||u(z)| e^{\phi(\psi(z))} e^{-\phi(z)}\right\|_{L^{\infty}} .
$$

Proof. For each $w \in \mathbb{C}^{n}$, set $f_{w}(z)=K_{\phi, w}(z) e^{-\phi(w)}$. It is clear that $f_{w} \in \mathcal{F}_{\phi}^{p}$, for $p \in(0, \infty]$, and $\left\|f_{w}\right\|_{p, \phi} \leq C$ for some constant $C>0$. Then,

$$
\begin{aligned}
\left\|u C_{\psi}\left(f_{w}\right)\right\|_{\infty, \phi} & =\sup _{z \in \mathbb{C}^{n}}\left|u(z) f_{w}(\psi(z))\right| e^{-\phi(z)} \\
& \geq|u(z)|\left|K_{\phi, w}(\psi(z))\right| e^{-\phi(w)} e^{-\phi(z)} .
\end{aligned}
$$

Therefore, for any $w, z \in \mathbb{C}^{n}$, we have

$$
|u(z)|\left|K_{\phi, w}(\psi(z))\right| e^{-\phi(w)} e^{-\phi(z)} \leq\left\|u C_{\psi}\right\|_{\infty, \phi}\left\|f_{w}\right\|_{p, \phi} .
$$

Set $w=\psi(z)$, we get

$$
|u(z)|\left|K_{\phi, \psi(z)}(\psi(z))\right| e^{-\phi(\psi(z))} e^{-\phi(z)} \leq C\left\|u C_{\psi}\right\|_{\infty, \phi} .
$$

Using (1) and (2), we get $\left|K_{\phi, \psi(z)}(\psi(z))\right| \simeq e^{2 \phi(\psi(z))}$, this implies for some positive constant $C_{1}$, we have

$$
C\left\|u C_{\psi}\right\|_{\infty, \phi} \geq C_{1}|u(z)| e^{2 \phi(\psi(z))} e^{-\phi(\psi(z))} e^{-\phi(z)} .
$$


Hence, taking supremum over $z \in \mathbb{C}^{n}$, we get

$$
\left\|u C_{\psi}\right\|_{\infty, \phi} \geq C_{2}\left\||u(z)| e^{\phi(\psi(z))} e^{-\phi(z)}\right\|_{L^{\infty}} .
$$

For the converse, by Proposition 2.3 of [13], for any $f \in \mathcal{F}_{\phi}^{p}$ and for some constant $C_{3}$, we have

$$
|f(z)| \leq C_{3} e^{\phi(z)}\|f\|_{p, \phi}
$$

This implies

$$
\begin{aligned}
\left\|u C_{\psi}(f)\right\|_{\infty, \phi} & =\sup _{z \in \mathbb{C}^{n}}|u(z) f(\psi(z))| e^{-\phi(z)} \\
& \leq C_{3}\|f\|_{p, \phi} \sup _{z \in \mathbb{C}^{n}}|u(z)| e^{\phi(v(z))} e^{-\phi(z)} .
\end{aligned}
$$

Therefore,

$$
\left\|u C_{\psi}\right\|_{\infty, \phi} \leq C_{3} \sup _{z \in \mathbb{C}^{n}}|u(z)| e^{\phi(\psi(z))} e^{-\phi(z)},
$$

which gives the desired result.

Theorem 2.2. Let $0<p \leq \infty$, let $\psi$ be an entire self-map of $\mathbb{C}^{n}$, and let $u$ be an entire function of $\mathbb{C}^{n}$. Then the operator $u C_{\psi}: \mathcal{F}_{\phi}^{p} \rightarrow \mathcal{F}_{\phi}^{\infty}$ is compact if and only if it is bounded and

$$
\lim _{|\psi(z)| \rightarrow \infty}|u(z)| e^{\phi(\psi(z))} e^{-\phi(z)}=0 .
$$

Proof. First, assume that $u C_{\psi}$ is compact. For each $w \in \mathbb{C}^{n}$, set $f_{w}(z)=K_{\phi, w}(z) e^{-\phi(w)}$. It is known that the sequence $\left\{f_{w}\right\}$ converges to zero uniformly on compact subsets of $\mathbb{C}^{n}$ as $|w| \rightarrow \infty$. By Lemma 1.1, compactness of $u C_{\psi}$ implies that

$$
\left\|u C_{\psi} f_{w}\right\|_{\infty, \phi} \rightarrow 0 \text { as }|w| \rightarrow \infty .
$$


Now suppose that there exists a sequence $\left\{z_{j}\right\} \in \mathbb{C}^{n}$ such that $\left|\psi\left(z_{j}\right)\right| \rightarrow \infty$ as $j \rightarrow \infty$. Hence, from (5), for some positive constant $C$, we get

$$
\begin{aligned}
C \limsup _{j \rightarrow \infty}\left|u\left(z_{j}\right)\right| e^{\phi\left(\psi\left(z_{j}\right)\right)} e^{-\phi\left(z_{j}\right)} & \leq \limsup _{j \rightarrow \infty}\left\|u C_{\psi} f_{\psi}\left(z_{j}\right)\right\|_{\infty, \phi} \\
& =0,
\end{aligned}
$$

which gives the desired result, $\lim _{|\psi(z)| \rightarrow \infty}|u(z)| e^{\phi(\psi(z))} e^{-\phi(z)}=0$.

Second, suppose that $u C_{\psi}$ is bounded and condition (4) holds. Let $\left\{f_{j}\right\}$ be a bounded sequence of functions in $\mathcal{F}_{\phi}^{p}$, say bounded by $C$, and converges uniformly to zero on compact subsets of $\mathbb{C}^{n}$ as $j \rightarrow \infty$. Using our hypothesis, condition (4), for each $\epsilon>0$, there exists $r>0$ such that for all $|\psi(z)|>r$

$$
|u(z)| e^{\phi(\psi(z))} e^{-\phi(z)}<\epsilon
$$

From (6) and Proposition 2.3 of [13], for all $|\psi(z)|>r$ and some $C_{1}>0$, we obtain

$$
\begin{aligned}
\left|u C_{\psi}\left(f_{j}(z)\right)\right| e^{-\phi(z)} & =|u(z)|\left|f_{j}(\psi(z))\right| e^{-\phi(z)} \\
& \leq C_{1}\left\|f_{j}\right\|_{p, \phi}|u(z)| e^{\phi(\psi(z))} e^{-\phi(z)} \\
& \leq \epsilon C C_{1} .
\end{aligned}
$$

Note that if $f(z)=1$, then $\|f\|_{p, \phi}=1$. It follows, by the boundedness of $u C_{\psi}$, that is, $u \in \mathcal{F}_{\phi}^{\infty}$. Hence, if $|\psi(z)| \leq r$, we get

$$
\left|u(z) f_{j}(\psi(z))\right| e^{-\phi(z)} \leq\|u\|_{\infty, \phi} \sup _{|w| \leq r}\left|f_{j}(w)\right| \rightarrow 0 \text { as } j \rightarrow \infty .
$$


From (7) and (8), we get

$$
\begin{aligned}
\lim _{j \rightarrow \infty}\left\|u C_{\psi}\left(f_{j}\right)\right\|_{\infty, \phi} & =\lim _{j \rightarrow \infty} \sup _{z \in \mathbb{C}^{n}}\left|u(z) f_{j}(\psi(z))\right| e^{-\phi(z)} \\
& =0 .
\end{aligned}
$$

Hence, by Lemma 1.1, we get the compactness of $u C_{\psi}$. This completes the proof.

Theorem 2.3. Let $0<p \leq \infty$, let $\psi$ be an entire self-map of $\mathbb{C}^{n}$, and let $u$ be an entire function of $\mathbb{C}^{n}$. Then the operator $u C_{\psi}: \mathcal{F}_{\phi}^{p} \rightarrow \mathcal{F}_{\phi}^{\infty, 0}$ is compact if and only if it is bounded and

$$
\lim _{|z| \rightarrow \infty}|u(z)| e^{\phi(\psi(z))} e^{-\phi(z)}=0
$$

Proof. Assume that the operator $u C_{\psi}$ is compact from $\mathcal{F}_{\phi}^{p}$ into $\mathcal{F}_{\phi}^{\infty, 0}$. Since $\mathcal{F}_{\phi}^{\infty, 0}$ is a proper Banach subspace of $\mathcal{F}_{\phi}^{\infty}$, we get that $u C_{\psi}$ is compact from $\mathcal{F}_{\phi}^{p}$ to $\mathcal{F}_{\phi}^{\infty}$. Hence, by Theorem 2.2, condition (4) holds. Therefore, for each $\epsilon>0$, there exists $r>0$ such that for all $|\psi(z)|>r$

$$
|u(z)| e^{\phi(\psi(z))} e^{-\phi(z)}<\epsilon .
$$

On the other hand, if $f(z)=1$, we get $u \in \mathcal{F}_{\phi}^{\infty, 0}$, so $\lim _{|z| \rightarrow \infty}|u(z)| e^{-\phi(z)}=0$. Hence there exists $r_{1}>0$ such that $|u(z)| e^{-\phi(z)}<\epsilon$ for $|z|>r_{1}$. Now if $|\psi(z)| \leq r$ and $|z|>r_{1}$, then there exists a constant $C>0$ such that

$$
|u(z)| e^{\phi(\psi(z))} e^{-\phi(z)}<\epsilon C,
$$

where the last inequality holds, because if $|\psi(z)| \leq r$ then $\phi(\{\psi(z):|\psi(z)| \leq r\})$ is compact and so bounded set in $\mathbb{C}^{n}$. Therefore, from (10) and (11), we get 


$$
\lim _{|z| \rightarrow \infty}|u(z)| e^{\phi(\psi(z))} e^{-\phi(z)}=0 .
$$

For the converse, assume that condition (9) holds. Then for each $\epsilon>0$, there exists $r_{2}>0$ such that

$$
|u(z)| e^{\phi(\psi(z))} e^{-\phi(z)}<\epsilon,
$$

for all $|z|>r_{2}$. Now, let $\left\{f_{j}\right\}$ be a uniformly bounded, say by $C^{*}$, sequence of functions in $\mathcal{F}_{\phi}^{p}$ and converges uniformly to zero on compact subsets of $\mathbb{C}^{n}$ as $j \rightarrow \infty$. We processed to show that $u C_{\psi}$ is compact, by Lemma 1.1, it is enough to show $\lim _{j \rightarrow \infty}\left\|u C_{\psi}\left(f_{j}\right)\right\|_{\infty, \phi}=0$. By Proposition 2.3 of [13] and inequality (12), for all $|z|>r_{2}$, we obtain

$$
\left|u C_{\psi}\left(f_{j}(z)\right)\right| e^{-\phi(z)} \leq \sup _{j \geq 1}\left\|f_{j}\right\|_{p, \phi}|u(z)| e^{\phi(\psi(z))} e^{-\phi(z)} \leq \epsilon C^{*} .
$$

Now if $|z| \leq r_{2}$, then the set $\psi\left(\left\{z:|z| \leq r_{2}\right\}\right)$ is compact and so bounded, say by $r_{3}$. Then,

$$
\begin{aligned}
\left|u C_{\psi}\left(f_{j}(z)\right)\right| e^{-\phi(z)} & =|u(z)| e^{-\phi(z)} f_{j}(\psi(z)) \\
& \leq\|u\|_{\infty, \phi} \sup _{|\psi(z)| \leq r_{3}} f_{j}(\psi(z)) \rightarrow 0 \text { as } j \rightarrow \infty .
\end{aligned}
$$

Hence, from (13) and (14), we get

$$
\begin{aligned}
0 & =\lim _{j \rightarrow \infty} \sup \left|u C_{\psi}\left(f_{j}(z)\right)\right| e^{-\phi(z)} \\
& =\lim _{j \rightarrow \infty}\left\|u C_{\psi}\left(f_{j}\right)\right\|_{\infty, \phi}
\end{aligned}
$$

this gives the desired result. 
Remark 2.4. In the previous Theorems 2.1 and 2.2, we used the test function $f_{w}(z)=K_{\phi, w}(z) e^{-\phi(w)}$. We have seen that $\left\|f_{w}\right\|_{p, \phi}<\infty$, for $0<p \leq \infty$, that is $f_{w} \in \mathcal{F}_{\phi}^{p}$. Now, for positive constants $\theta$ and $C$, we have

$$
\begin{aligned}
\left\|f_{w}\right\|_{\infty, 0, \phi} & =\lim _{|z| \rightarrow \infty}\left|f_{w}(z)\right| e^{-\phi(z)} \\
& =\lim _{|z| \rightarrow \infty}\left|K_{\phi, w}(z)\right| e^{-\phi(w)} e^{-\phi(z)} \\
& \leq C \lim _{|z| \rightarrow \infty} e^{-\theta|z-w|}=0 .
\end{aligned}
$$

This gives $f_{w} \in \mathcal{F}_{\phi}^{\infty, 0}$. Moreover, we know that the space $\mathcal{F}_{\phi}^{\infty, 0}$ contains the space $\mathcal{F}_{\phi}^{p}$ for all $0<p<\infty$. Therefore, if we replace the space $\mathcal{F}_{\phi}^{p}$ with a larger space $\mathcal{F}_{\phi}^{\infty, 0}$ in the previous three theorems, these theorems are still true and the conditions remain unchanged.

\section{References}

[1] B. Carswell, B. D. MacCluer and A. Schuster, Composition operators on the Fock space, Acta Sci. Math. (Szeged) 69 (2003), 871-887.

[2] R. Cho and K. Zhu, Fock-Sobolev spaces and their Carleson measures, J. Funct. Anal. 263 (2012), 2483-2506.

[3] M. Christ, On the $\bar{\partial}$ equation in weighted $L^{2}$ norms in $C^{1}$, J. Geom. Anal. 3 (1991), 193-230.

[4] C. Cowen and B. D. MacCluer, Composition Operators on Spaces of Analytic Functions, CRC Press, Boca Raton, 1995.

[5] Z. Čučković and R. Zhao, Weighted composition operators between different weighted Bergman spaces and different Hardy spaces, Ill. J. Math. 51 (2007), 479-498.

[6] Z. Čučković and R. Zhao, Weighted composition operators on the Bergman space, J. Lond. Math. Soc. 70 (2004), 499-511. 
[7] H. Delin, Pointwise estimates for the weighted Bergman projection kernel in $\mathbb{C}^{n}$, using a weighted $L^{2}$ estimate for the $\bar{\partial}$ equation, Ann. Inst. Fourier (Grenoble) 48 (1998), 967-997.

[8] P. Duren and A. Schuster, Bergman Spaces, American Mathematical Society, 2004.

[9] H. Hedenmalm, B. Korenblum and K. Zhu, Theory of Bergman Spaces, SpringerVerlag, New York, 2000.

[10] B. D. MacCluer and J. H. Shapiro, Angular derivatives and compact composition operators on the Hardy and Bergman spaces, Canad. J. Math. 38 (1986), 878-906.

[11] T. Mengestie, Carleson type measures for weighted Fock-Sobolev spaces, Complex Anal. Oper. Theory 8 (2014), 1225-1256.

[12] W. Al-Rawashdeh, Essential norm of weighted composition operators on BergmanFock spaces, preprint.

[13] A. Schuster and D. Varolin, Toeplitz operators and Carleson measures on generalized Bergman-Fock spaces, Integr. Equ. Oper. Theory 72 (2012), 363-392.

[14] J. H. Shapiro, Composition Operators and Classical Function Theory, Universitext: Tracts in Mathematics, Springer-Verlag, New York, 1993.

[15] S. Stević, Weighted composition operators between Fock-type spaces in $\mathbb{C}^{N}$, Appl. Math. Comput. 215 (2009), 2750-2760.

[16] Y. Tung, Fock Spaces, Ph.D. Dissertation, University of Michigan, 2005.

[17] S. Ueki, Weighted composition operators on some function spaces of entire functions, Bull. Belg. Math. Soc. Simon Stevin 17 (2010), 343-353.

[18] S. Ueki, Weighted composition operator on the Bergman-Fock spaces, Int. J. Mod. Math. 3 (2008), 231-243.

[19] S. Ueki, Weighted composition operator on the Fock space, Proc. Amer. Math. Soc. 135 (2007), 1405-1410.

[20] K. Zhu, Analysis on Fock Spaces, Springer-Verlag, New York, 2012.

[21] K. Zhu, Operator Theory in Function Spaces, American Mathematical Society, 2007.

[22] K. Zhu, Spaces of Holomorphic Functions in the Unit Ball, Springer-Verlag, New York, 2005. 